\title{
Epidemiology and Phenotypic Characteristics of Dual Molecular Diagnosis Cases in Skeletal Abnormality
}

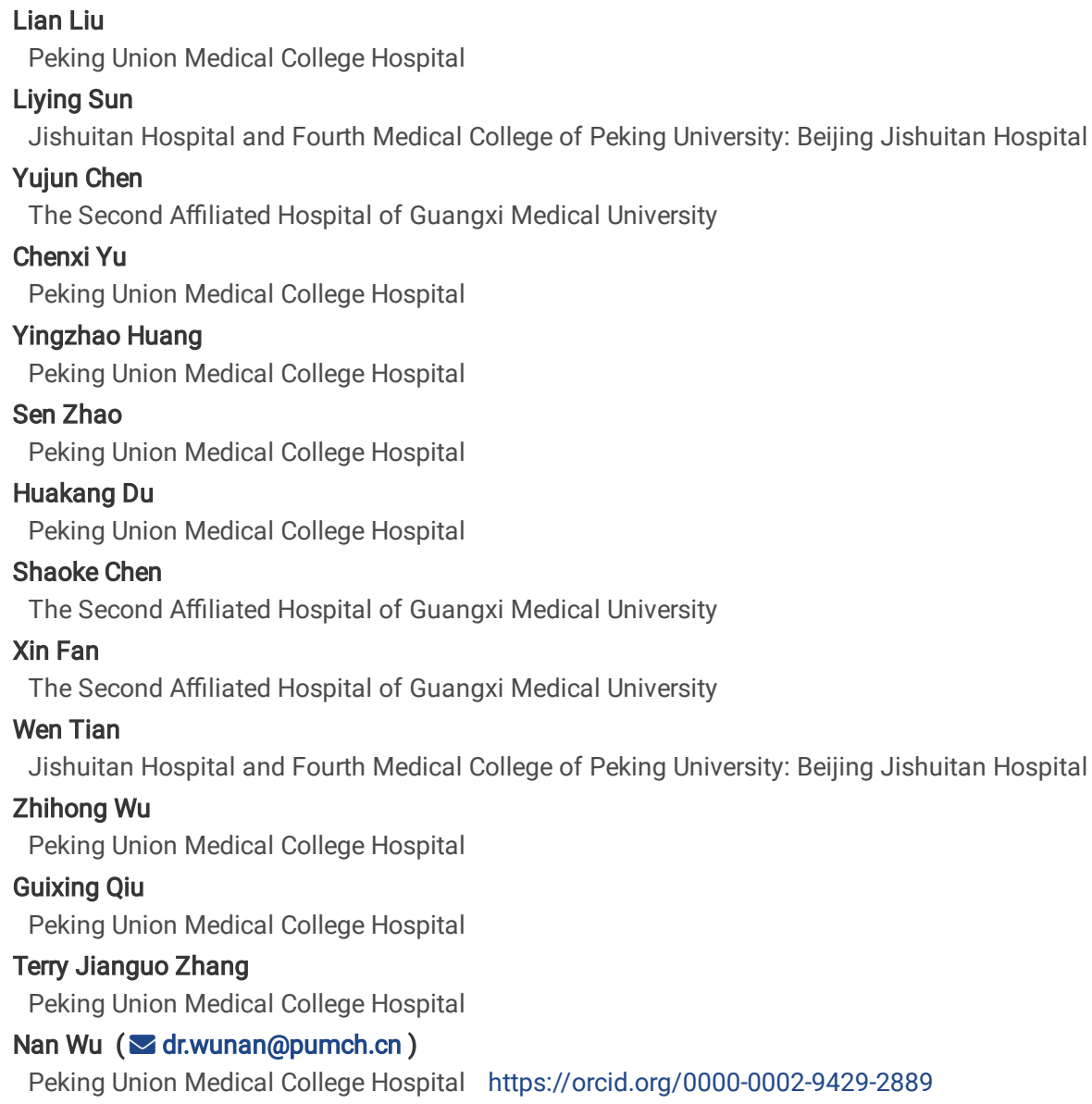

\section{Research Article}

Keywords: Skeletal abnormality, Phenotypic characteristics, Dual molecular diagnosis

Posted Date: July 30th, 2021

DOI: https://doi.org/10.21203/rs.3.rs-717820/v1

License: (a) (i) This work is licensed under a Creative Commons Attribution 4.0 International License. Read Full License 


\begin{abstract}
Background

Skeletal abnormality is a heterogeneous group of disorders that affects the composition and structure of bone and cartilage. In our previous studies, we have revealed that a substantial proportion of cases with early-onset scoliosis could be explained by monogenic disorders such as Marfan syndrome and EhlersDanlos syndrome. More recently complex phenotypes caused by more than one genetic defect (i.e., dual molecular diagnosis) have also been reported in skeletal abnormalities. To explore the molecular epidemiology and phenotypic characteristics of dual diagnosis in skeletal abnormalities, we described cases with dual molecular diagnosis from the Deciphering disorders Involving Scoliosis and COmorbidities (DISCO) study.
\end{abstract}

\title{
Results
}

In total, 1108 patients with skeletal abnormality from the DISCO study underwent Exome Sequencing. We identified eight probands with dual molecular diagnosis, including three (0.7\%) from individuals with early-onset scoliosis (EOS), three (0.5\%) from individuals with short stature, and two ( $2 \%$ ) from individuals with congenital hand/foot deformity (CHFA). Other skeletal abnormalities observed in these individuals included bone fracture and interphalangeal joint contracture. All the eight probands have dual diagnosis of two autosomal dominant (AD) diseases. A total of 16 variants in 12 genes were identified. A substantial rate (5 of 10) of the identified causal variants were of de novo origin. The frequently observed molecular diagnoses (observed in more than one patient) include Osteogenesis Imperfecta Type I (COL 1A1, MIM:166200), Neurofibromatosis, Type I (NF1, MIM:162200) and Marfan Syndrome (FBN1, MIM:154700). These patients with dual molecular diagnosis often present blended phenotypes of two diseases, which significantly complicate their diagnostic process.

\section{Conclusions}

This study revealed the molecular epidemiology and complex diagnostic odyssey of dual molecular diagnosis through analyzing the clinical traits of skeletal abnormalities in eight patients.

\section{Background}

Skeletal abnormality is a heterogeneous group of disorders that affects the composition and structure of bone and cartilage (1). Phenotypes involved in skeletal abnormality include short stature, scoliosis, joint dislocation, limb deformities, and bone density changes etc. According to the tenth version of the Nosology and Classification of Genetic Skeletal Disorders, there are 461 different diseases that are classified into 42 groups based on their clinical, radiographic, and/or molecular findings (2). Genetic factors play an important role during the pathogenesis of skeletal abnormalities. In our previous studies, we have revealed that a substantial proportion of cases with early-onset scoliosis could be explained by monogenic disorders such as Marfan syndrome (MIM:154700) and Ehlers-Danlos syndrome (MIM:130000) (3).

In addition to monogenic conditions, complex phenotypes caused by more than one genetic defect (i.e. dual molecular diagnosis) have also been reported in skeletal abnormalities. For example, Aggarwal et al. reported a fetus with complex joint dislocations and congenital scoliosis who was detected to be double heterozygote for putatively pathogenic Fibrillin-1 ( $F B N 1$ ) and Fibrillin-2 (FBN2) variants (4). Tang et al. identified a patient with pathogenic variants in both FBN1 and protein tyrosine phosphatase, non-receptor type 11 gene (PTPN11), resulting in combined phenotypes of Marfan syndrome (MIM:154700) and LEOPARD syndrome (MIM:151100) (5).

The co-existence of two Mendelian conditions in one individual brings challenges to the diagnosis and clinical management. The precise diagnosis of such conditions often relies on comprehensive genetic testing such as exome sequencing (ES) (6-8). Thus far, systematic investigations of dual molecular diagnosis have been performed in neurodevelopmental disorders $(9,10)$, genetic muscle diseases (11), endocrine dysfunction (10) and so on. However, the molecular epidemiology and phenotypic characteristics of dual diagnosis in skeletal abnormality is still elusive.

Here, we describe eight cases with dual molecular diagnosis from the Deciphering disorders Involving Scoliosis and COmorbidities (DISCO) study. We also report the phenotypic characteristics of these patients and clinical relevance for certain genes and Mendelian syndromes.

\section{Results}

\section{Molecular epidemiology}

In total, 1108 patients with skeletal abnormality from the DISCO study underwent ES. We identified eight probands with dual molecular diagnosis, including three $(0.7 \%)$ from individuals with EOS, three $(0.5 \%)$ from individuals with short stature, and two (2\%) from individuals with CHFA (Table 1$)$. All the eight probands have dual diagnosis of two autosomal dominant (AD) diseases. A total of 16 variants in 12 genes were identified. A substantial rate (5 of 10) of the identified causal variants were of de novo origin. The frequently observed molecular diagnoses (observed in more than one patient) include Osteogenesis Imperfecta Type I (COL1A1, MIM:166200), Neurofibromatosis, Type I (NF1, MIM:162200) and Marfan Syndrome (FBN1, MIM:154700).

\section{The complex clinical features of patients with dual molecular diagnosis}

Patients with dual molecular diagnosis often present blended phenotypes of two diseases, which significantly complicate their diagnostic odysseys. Here we report the detailed clinical characteristics of these patients to demonstrate the effect dual molecular diagnosis on the diagnosis of skeletal abnormality. 


\section{Case 1}

Patient DISCO-SCO2003P1972 was a 7-year-old boy with early-onset scoliosis (Figure 1.A, Supplementary Figure 1.A-B). At 2 years old, he was diagnosed with congenital dislocation of hip joint (Table 1) and underwent a surgical reduction. Meantime, a slight scoliosis was found during the hospitalization. At 7 years old, congenital scoliosis with segmentation failure of T10-L1 was identified through CT examination (Figure 1.A, Table 1). Physical examination showed disproportionate ocular hypertelorism and intellectual disability (Figure 1.A, Table 1). ES revealed a pathogenic heterozygous nonsense variant (c.2649G>A p.Trp883Ter) in FBN1 (Table 1), which is associated with Marfan syndrome (MIM:154700) (3). Consistently, the proband also presented Marfan syndromerelated phenotypes such as mitral valve prolapse, mild arachnodactyly and scoliosis. This variant was inherited from his mother, who had severe scoliosis, arachnodactyly and long slender limbs (Figure 1.A). Through further analysis of the exome data, a de novo variant in POGZ (c.1180_1181del p.Met394ValfsTer9) (Table 1) was found in the proband. This variant was previously reported and was associated with White-Sutton syndrome (WHSUS) (MIM:616364) characterized by intellectual disability, ocular abnormalities and skeletal deformities (12), which largely overlapped with the phenotypes of this patient. Therefore, we suggested that the complex phenotypes of this patient could be explained by a combined effect of variants in $P O G Z$ and FBN1.

\section{Case 2}

In case 2, the proband (DISCO-SC01908P0067) was first referred to the clinic due to scoliosis at the age of fifteen years. The patient was clinically diagnosed as adolescent idiopathic scoliosis (AIS) (Supplementary Figure 1.C), i.e., scoliosis with unknown etiology (Table 1). He also presented pectus carinatum (Table 1). He experienced fracture twice at the age of 10 and 13. ES revealed that he carried two pathogenic variants, including a de novo variant in COL1A1 (c.1081C>T p.Arg361Ter) and a maternally inherited variant in FBN1 (c.1453C>T p.Arg485Cys) (Table 1). These two reported variants led to dual molecular diagnosis consisting of osteogenesis imperfecta type I and Marfan syndrome $(13,14)$. Although reported patients with osteogenesis imperfecta type I often present short stature (15), the stature of this patient is relatively high tall (Height: $185 \mathrm{~cm}$ ) (Figure 1.B , Table 1), which might be related with his second diagnosis of Marfan syndrome. Although FBN1 variant has been reported and considered as a pathogenic variant (14), no other features of Marfan Syndrome such as dolichostenomelia, arachnodactyly, joint laxity, velvety skin, ectopia lentis and cardiovascular manifestations were identified. This patient exemplified the apparently contrary effects of two monogenic disease on the same trait (height in this case).

\section{Case 3}

Patient DISCO-PCT2007P0019 was an 8-year-old girl. The proband suffered from right tibia fracture at the age of 2 years. At around the age of 6 years, the patient developed razorback deformity and unbalanced shoulder, accompanied with mild scoliosis. A progressive scoliosis was observed in the next 2 years (Supplementary Figure 1.D-E). A series of blended clinical phenotypes, such as blue sclera, cafe-au-Lait macules were observed as well (Figure 1.C, Table 1). We found vertebral malformations (T6, T7) in this patient (Figure 1.C) through radiographic examinations. Her father and younger brother presented with blue sclera but without any history of bone fracture. ES identified the one pathogenic variant in NF1 (c.2307del p.Thr770LeufsTer21) and another pathogenic variant in $\operatorname{COL} 1 A 1$ (c. 2028+4A>G) (Table 1), both transmitted from her father. The NF1 variant could lead to a frameshift of NF1 (Table 1) and thus a loss-offunction effect, which is associated with neurofibromatosis type I and could explain the cafe-au-Lait macules in this patient (Figure 1.C, Table 1). The COL $1 A 1$ splicing variant (c.2028+4A>G) has been previously described to cause osteogenesis imperfecta (16), which could explain the recurrent bone fracture history in this patient. Interestingly, both neurofibromatosis type I and osteogenesis imperfecta are associated with scoliosis but with incomplete penetrance $(15,17)$. Therefore, the scoliotic phenotype in this patient might be caused by the synergistic effects of the dual molecular diagnosis.

\section{Case 4}

In this case, we reported a 6-year-old boy (DISCO-WAX677) with short stature and craniofacial abnormalities including depressed nasal bridge and long philtrum (Table 1). Then the patient was detected to be double heterozygote for putatively pathogenic ANKRD11 (c.2508dup p.Leu837ThrfsTer81) and COL11A1 (c.1180_1181del p.Met394ValfsTer9) variants on WES (Table 1). The ANKRD11 and COL11A1 variants were confirmed as de novo and paternal status, separately (Table 1). The clinical findings of the present case are compared with the reported phenotypes of KBG syndrome (MIM:148050) and Marshall syndrome (MIM:154780), caused by ANKRD11 and COL11A1 variants, respectively. A comparison between the findings in the patient and reported phenotypes of KBG and Marshall syndrome indicated overlapping clinical features in this case.

\section{Case 5}

This patient (DISCO-AX168) was an 8-year-old girl with short stature and developmental delay. She also presented widespread cafe-au-Lait macules and lumbar scoliosis (Figure 1.D, Table 1). Additionally, her father $(162 \mathrm{~cm})$ and mother $(140 \mathrm{~cm})$ were both presented with short stature. Brain magnetic resonance (MR) was performed and abnormality of the cerebral white matter was identified. According to previously study (18), combined with MR results and family history, the patient was diagnosed with idiopathic short stature (ISS). Subsequently, the girl was sequenced and two pathogenic variants (Table 1), including a splicing variant in the NF1 gene (c.6705-1G>A) and a frameshift variant in the GL/2 gene (c.1189del p.Val397CysfsTer124) were identified. Variants in GLI2 have been shown to cause short stature, abnormal development of brain structures, hypopituitarism and facial dysmorphism in Culler-Jones syndrome (MIM:615849)(19). We suggested this patient's presentation represents a mixture of distinct phenotypes, i.e., Cafe-au-Lait spots for NF type 1 (NF1, MIM:162200) and short stature for Culler-Jones syndrome (MIM:615849). 


\section{Case 6}

Case 6 (DISCO-AX282) was a 12-year-old girl with short stature (Table 1). She also presented mild scoliosis, low posterior hairline, hyper pigmentation and webbed neck (Figure 1.E, Table 1). ES was performed to find the potential molecular etiology. A pathogenic nonsense variant in TP63 (c.109C>T p.Arg37Ter) and another pathogenic missense variant in PTPN11 (c.1510A>G p.Met504Val) were identified (Table 1). On the basis of published studies (20, 21), as well as phenotypes in this patient, we concluded this patient carried variants in both TP63 and PTPN11, resulting in a blended phenotype of Rapp-Hodgkin syndrome (MIM: 129400) and Noonan syndrome (MIM: 163950). Overlapping phenotypes (especially craniofacial malformations) of these two syndromes make the diagnosis challenging if solely based on clinical features.

\section{Case 7}

A 3-year-old proband (DISCO-RDD2001P0005) presented at birth with widespread interphalangeal joint contractures of hand (Figure 1.F) and atrial septal defect (Table 1). His father also experienced hand contracture deformities during his childhood. ES revealed a heterozygous variant (c.3437A>G p.Tyr1146Cys) in FBN2 gene and a heterozygous ANKRD11 variant (c.3024_3025del p.Lys1009GlyfsTer8), both with unknown origin (Table 1). The FBN2 variant could lead to Beals syndrome (Congenital contractual arachnodactyly) (MIM:121050), which is characterized by hand interphalangeal joint contractures $(22,23)$; The ANKRD11 mutation could cause KBG syndrome, which could explain the atrial septal defect in this patient (24). Therefore, this patient present mixed phenotypes of two distinct Mendelian disorders.

\section{Case 8}

This case is a 5-year-old male (DISCO-P19009402) with complex phenotypes. He presented congenital syndactyly of hand and foot (Supplementary Figure 1.F-G), cloverleaf skull, orbital hypertelorism, proptosis and midfacial hypoplasia (Table 1). He also had a history of hyperthermia during general anesthesia. ES identified a de novo missense variant (c.755C>G p.Ser252Trp) in FGFR2 gene and a paternally inherited frameshift variant in $R Y R 1$ gene (c.12788_12793dup p.Glu4263_Gly4264dup) (Table 1). The FGFR2 variant occurred in a known Apert syndrome hotspot (25). As reported, Apert syndrome was characterized by craniosynostosis, proptosis, midfacial hypoplasia and severe syndactyly of the hands and feet (25), which are concordant with the phenotypes of this patient. Pathogenic RYR1 variants are associated with malignant hyperthermia susceptibility 1(MHS 1) (MIM:145600) (26), which could explain the hyperthermia history in the patient.

Table 1

Summary of the clinical and molecular findings of studied subjects

\section{Discussion}

The development of ES has significantly improved diagnostic yield of rare disease in recent years. Through analyzing the ES data from 7374 patients, 2182 independent molecular diagnoses were reported by Posey et al. $(6,8)$. A molecular diagnosis involving a mendelian disease was reported for 2076 patients (28.2\%) and two or more molecular diagnoses were reported for 101 patients $(4.9 \%)(6,8)$. Additionally, copy number variants (CNVs) and single nucleotide variants (SNVs) as a part of multiple diagnoses was reported in 12 of 101 patients (11.9\%) by Posey et al (6). Yang et al. found ES identified the underlying genetic defect in $25 \%$ of consecutive patients referred for evaluation of a possible genetic condition and $4.6 \%$ patients with blended phenotypes resulting from two single gene defects (7).

In our study, most probands (5 out of 8 ) had family members for at least one of the diseases, consistent with the report by Balci et al (27). All the 8 probands have variants in $\mathrm{AD}$ disease genes and de novo variants were the most common pathogenic variants origin and accounted for $40 \%$ of pathogenic variants in 8 families. These results are consistent with previously published articles, which revealed that AD was the most frequently seen inheritance mode among patients with multiple diagnoses (8). There're two types of effect caused by the mutual interaction of two pathogenic variants. One was called "synergistic effect", which means that the combination of two mutational genes in patients would lead to more severe phenotypes. For instance, Xe et al. revealed a patient with one variant in CSNK2A1 and another in TRPS1, which resulted in a dual molecular diagnosis of tricho-rhino-phalangeal syndrome type I (TRPS I) and Okur-Chung neurodevelopmental syndrome (OCNDS). These two syndromes are both associated with short stature. Notably, compared with other patients with single diagnosis as well as his parents, this patient has a more severe degree of short stature (9). Moreover, Ye et al. reported a familial case with 13 patients affected by osteogenesis imperfecta (OI) type I, short stature and advanced bone age, with or without early-onset osteoarthritis and/or osteochondritis dissecans (SSOAOD). The proband was found to have two variants in two genes: COL 1A1 and ACAN. After comprehensive analysis of the height within the family who carried either or both of the variants, this study discovered a synergistic effect that the patients with two variants present the most severe form of short stature (28). These manifestations resembled clinical presentations of one of our patients (DISCO-SCO2003P1972). The kid was presented with a segmentation failure of T10-L1 of spinal vertebrae, which was extremely unusual phenotype of classical Marfan Syndrome. Additionally, a severe scoliosis, which presented with a thoracolumbar curve $\left(\mathrm{Cobb}>80^{\circ}\right)$, were observed in the kid. We concluded that the $P O G Z$ and $F B N 1$ variants both contributed to the skeletal abnormality in this patient. However, in some patients who were diagnosed with dual molecular diagnosis, certain phenotypes may be opposite to that caused by a single genetic mutation. We proposed that these phenomena were caused by an antagonistic effect. Tang et al. reported a patient with variants in both FBN1 and PTPN11 genes with combined phenotypes of Marfan (MIM:154700) and LEOPARD (MIM:151100) syndromes (5). However, this patient presented with short stature, other than classical tall stature of Marfan syndrome. We suppose that this phenomenon probably caused 


\begin{tabular}{|c|c|c|c|c|c|c|c|c|c|c|c|}
\hline $\begin{array}{l}\text { Case } \\
\text { Number }\end{array}$ & Case ID & Age & Sex & Inheritance & Gene & $\begin{array}{l}\text { Clinical } \\
\text { diagnosis }\end{array}$ & $\begin{array}{l}\text { Molecular } \\
\text { diagnosis }\end{array}$ & Zygosity & $\begin{array}{l}\text { Transcript } \\
\text { numbers }\end{array}$ & Origin & $\begin{array}{l}\text { Vari: } \\
\text { type }\end{array}$ \\
\hline Case 1 & SC02003P1972 & 7 & M & $A D$ & POGZ & CS II & $\begin{array}{l}\text { White-Sutton } \\
\text { Syndrome }\end{array}$ & Het & NM_015100.3 & de novo & Fran \\
\hline Case 1 & SC02003P1972 & 7 & M & $A D$ & FBN1 & CS II & Marfan Syndrome & Het & NM_000138.4 & maternal & Non: \\
\hline Case 2 & SC01908P0067 & 18 & M & $A D$ & COL $1 A 1$ & AIS & $\begin{array}{l}\text { Osteogenesis } \\
\text { imperfecta }\end{array}$ & Het & NM_000088.3 & de novo & Stop \\
\hline Case 2 & SC01908P0067 & 18 & M & $A D$ & FBN1 & AIS & Marfan Syndrome & Het & NM_000138.4 & maternal & Miss \\
\hline Case 3 & РСТ2007Р0019 & 8 & $\mathrm{~F}$ & $A D$ & NF1 & NFS & $\begin{array}{l}\text { Neurofibromatosis, } \\
\text { Type } 1\end{array}$ & Het & NM_000267.3 & paternal & Fran \\
\hline Case 3 & РСТ2007Р0019 & 8 & $\mathrm{~F}$ & $A D$ & COL $1 A 1$ & NFS & $\begin{array}{l}\text { Osteogenesis } \\
\text { imperfecta }\end{array}$ & Het & NM_000088.3 & paternal & Splic \\
\hline Case 4 & WAX677 & 6 & M & $A D$ & ANKRD11 & GHD & KBG syndrome & Het & NM_013275.5 & de novo & Non: \\
\hline Case 4 & WAX677 & 6 & M & $A D$ & COL11A1 & GHD & $\begin{array}{l}\text { Marshall } \\
\text { syndrome }\end{array}$ & Het & NM_001854.3 & paternal & Fran \\
\hline Case 5 & AX168 & 8 & $\mathrm{~F}$ & $A D$ & NF1 & ISS & $\begin{array}{l}\text { Neurofibromatosis, } \\
\text { Type } 1\end{array}$ & Het & NM_000267.3 & NA & Splic \\
\hline Case 5 & AX168 & 8 & $\mathrm{~F}$ & $A D$ & GLI2 & ISS & $\begin{array}{l}\text { Culler-Jones } \\
\text { syndrome }\end{array}$ & Het & NM_005270.4 & NA & Fran \\
\hline Case 6 & AX282 & 12 & $\mathrm{~F}$ & $A D$ & TP63 & ISS & $\begin{array}{l}\text { Rapp-Hodgkin } \\
\text { Syndrome }\end{array}$ & Het & NM_003722.4 & NA & Non: \\
\hline Case 6 & AX282 & 12 & $\mathrm{~F}$ & $A D$ & PTPN11 & ISS & Noonan Syndrome & Het & NM_002834.3 & NA & Miss \\
\hline Case 7 & RDD2001P0005 & 2 & M & $A D$ & FBN2 & $\mathrm{CPC}$ & Beals Syndrome & Het & NM_001999.3 & paternal & Miss \\
\hline Case 7 & RDD2001P0005 & 2 & M & $A D$ & ANKRD11 & $\mathrm{CPC}$ & KBG Syndrome & Het & NM_013275.5 & de novo & Fran \\
\hline Case 8 & P19009402 & 4 & M & $A D$ & FGFR2 & Syndactyly & Apert Syndrome & Het & NM_000141.4 & de novo & Miss \\
\hline Case 8 & P19009402 & 4 & M & $A D$ & $R Y R 1$ & Syndactyly & $\begin{array}{l}\text { Malignant } \\
\text { Hyperthermia } \\
\text { Susceptibility } 1\end{array}$ & Het & NM_000540.2 & paternal & Fran \\
\hline
\end{tabular}

by the predominant influence of the PTPN11 mutation over the FBN1 mutation (5).

All the dual diagnosis condition in our study were identified through ES. However, the high expense of ES hinders it from being either a stand-alone or a firsttier diagnostic approach. Therefore, selection the most appropriate molecular diagnostic tool is important when ordering genetic testing. Single-gene tests should be recommended when the clinical features for a patient were typical for a specific disorder and the association between the disorder and a single gene was well-established (29). For example, TBX6-associated congenital scoliosis was characterized by simple hemi-/wedge-shaped vertebrae in the lower half of the spine (30-32). Furthermore, our previous study found that a novel de novo FBN1 variant could explain the Marfanoid-progeroid-lipodystrophy syndrome (MIM:616914) (33). Under these circumstances, single-gene testing will be more preferred. In contrast, in cases with complex phenotypes as shown in our examples, ES can be utilized as first line test which could shorten the diagnostic odyssey of the patients (29).

In conclusion, this study revealed the molecular epidemiology and complex diagnostic odyssey of dual molecular diagnosis through analyzing the clinical traits of bone abnormality in eight patients.

\section{Conclusions}

This study recruited eight patients with dual molecular diagnosis from DISCO study and described the clinical traits of skeletal abnormalities. We reported the molecular epidemiology and complex diagnostic odyssey of dual molecular diagnosis. 


\section{Study design}

This is a retrospective study which reports the clinical and genetic characteristics of a group of patients with dual molecular diagnosis.

\section{Subjects}

Cases with skeletal abnormality from the DISCO study (http://www.discostudy.org/) who underwent ES were included. The types of skeletal abnormality include early-onset scoliosis (EOS) ( $N=447)$, short stature ( $N=561)$, and congenital hand/foot anomaly (CHFA) ( $N=100)$. Deep phenotyping and radiological examinations including X-ray, computed tomography (CT), and magnetic resonance imaging (MRI) were performed on each patient as previously reported $(3,34)$, (Fan et al., Journal of Genetics and Genomics, 2021, in press). Written informed consent was obtained from every participant; if the participant was younger than 16 years old, written informed consent was obtained from their parents or legal guardians. The study was approved by the institutional review board of PUMCH (JS-2364), Jishuitan Hospital (201808-09) and the Second Affiliated Hospital of Guangxi Medical University (G-1-1).

\section{Exome sequencing and variant interpretation}

ES was performed on DNA extracted from blood of all 8 probands and their family members. The sequencing data were analyzed and annotated using an inhouse developed analytical pipeline, Peking Union Medical College Hospital Pipeline (PUMP) (35-37). All variants were presumed to be pathogenic were subjected to Sanger sequencing.

\section{Identification of dual molecular diagnosis}

Patients with more than one molecular diagnosis from the included patients were selected for analyses. Each molecular diagnosis was manually curated based on the pathogenicity of the variants and the Mendelian expectations for inheritance mode. The pathogenicity of the variants was evaluated according to the American College of Medical Genetics and Genomics (ACMG) guidelines (38). The Mendelian expectations for inheritance mode include autosomal dominant ( $A D$ ) inheritance, autosomal recessive (AR) inheritance and X-linked dominant/recessive (XLD/XLR) inheritance. For $A D / X L D$ traits, one heterozygous pathogenic/likely pathogenic variant is sufficient to establish a molecular diagnosis. For AR/XLR traits, one homozygous, one hemizygous or one pair of compound heterozygous pathogenic/likely pathogenic variants are required for a molecular diagnosis.

\section{Abbreviations}

COL 1A1: Collagen, Type I, Alpha-1; NF1: Neurofibromatosis, Type I; FBN1: Fibrilin-1; FBN2: Fibrillin-2; PTPN11: protein tyrosine phosphatase, non-receptor type 11 gene; ES: exome sequencing; EOS: early-onset scoliosis; WHSUS. White-Sutton syndrome; Gli2: GLI-Kruppel family member 2; ANKRD11: Ankyrin repeat domain-containing protein 11; FGFR2: Fibroblast growth factor receptor 2; RYR1:Ryanodine receptor 1; CSNK2A1:Casein kinase II, alpha subunit; TRPS1: trichorhinophalangeal syndrome type $\mathrm{I}$.

\section{Declarations}

\section{Ethics approval and consent to participate}

The study was approved by the institutional review board of PUMCH (JS-2364), Jishuitan Hospital (201808-09) and the Second Affiliated Hospital of Guangxi Medical University (G-1-1). All participants gave informed consent.

\section{Consent for publication}

Consent for publication was obtained from all participants.

\section{Availability of data and materials}

The datasets used and/or analyzed during the current study are available from the corresponding author on reasonable request.

\section{Competing interests}

The authors declare that they have no competing interests.

\section{Funding}


This research was funded in part by the Beijing Natural Science Foundation (JQ20032 to N.W., 7191007 to Z.W.), Capital's Funds for Health Improvement and Research (2020-4-40114 to N.W.), National Natural Science Foundation of China (82072391 to N.W., 81930068 and 81772299 to Z.W., 81772301 and 81972132 to G.Q., 81972037 to J.Z.), Tsinghua University-Peking Union Medical College Hospital Initiative Scientific Research Program, Non-profit Central Research Institute Fund of Chinese Academy of Medical Sciences (No. 2019PT320025), Center for Rare Diseases Research, Chinese Academy of Medical Sciences (Grant No. 2016ZX310174-4).

\section{Authors' contributions}

NW directed the project. NW, JZ and GQ designed the study. LL, LS and YC were involved in data management and statistical analysis and wrote the first draft of the manuscript. CY, YH, HD and SC gathered detailed clinical information for the study. XF, WT and SZ analyzed the data. ZW, GQ, JZ and NW supervised the data analysis, and reviewed and commented on all drafts. All other authors coordinated the study and critically revised the manuscript. The author(s) read and approved the final manuscript.

\section{Acknowledgments}

We would like to thank all the individuals involved in the study for their participation. We thank the nurses and doctors from the Department of Orthopedic Surgery of Peking Union Medical College Hospital, the Second Affiliated Hospital of Guangxi Medical University and Beijing Jishuitan Hospital for assistance with patient enrollment. We thank geneseeq inc. for exome sequencing technical support. We thank for ekitech Itd. (Beijing) for big data management and genetic data analysis.

\section{References}

1. Krakow D, Lachman RS, Rimoin DL. Guidelines for the prenatal diagnosis of fetal skeletal dysplasias. Genet Med. 2009;11(2):127-33.

2. Mortier GR, Cohn DH, Cormier-Daire V, Hall C, Krakow D, Mundlos S, et al. Nosology and classification of genetic skeletal disorders: 2019 revision. Am J Med Genet A. 2019;179(12):2393-419.

3. Zhao S, Zhang Y, Chen W, Li W, Wang S, Wang L, et al. Diagnostic yield and clinical impact of exome sequencing in early-onset scoliosis (EOS). J Med Genet. 2021;58(1):41-7.

4. Aggarwal S, Das Bhowmik A, Tandon A, Dalal A. Exome sequencing reveals blended phenotype of double heterozygous FBN1 and FBN2 variants in a fetus. Eur J Med Genet. 2018;61(7):399-402.

5. Tang S, Hoshida H, Kamisago M, Yagi H, Momma K, Matsuoka R. Phenotype-genotype correlation in a patient with co-occurrence of Marfan and LEOPARD syndromes. Am J Med Genet A. 2009;149A(10):2216-9.

6. Posey JE, Rosenfeld JA, James RA, Bainbridge M, Niu Z, Wang X, et al. Molecular diagnostic experience of whole-exome sequencing in adult patients. Genet Med. 2016;18(7):678-85.

7. Yang Y, Muzny DM, Reid JG, Bainbridge MN, Willis A, Ward PA, et al. Clinical whole-exome sequencing for the diagnosis of mendelian disorders. N Engl J Med. 2013;369(16):1502-11.

8. Posey JE, Harel T, Liu P, Rosenfeld JA, James RA, Coban Akdemir ZH, et al. Resolution of Disease Phenotypes Resulting from Multilocus Genomic Variation. N Engl J Med. 2017;376(1):21-31.

9. Xu S, Lian Q, Wu J, Li L, Song J. Dual molecular diagnosis of tricho-rhino-phalangeal syndrome type I and Okur-Chung neurodevelopmental syndrome in one Chinese patient: a case report. BMC Med Genet. 2020;21(1):158.

10. Jehee FS, de Oliveira VT, Gurgel-Giannetti J, Pietra RX, Rubatino FVM, Carobin NV, et al. Dual molecular diagnosis contributes to atypical Prader-Willi phenotype in monozygotic twins. Am J Med Genet A. 2017;173(9):2451-5.

11. Hannah-Shmouni F, Al-Shahoumi R, Brady LI, Wu L, Frei J, Tarnopolsky MA. Dual molecular diagnoses in a neurometabolic specialty clinic. Am J Med Genet A. 2020.

12. Assia Batzir N, Posey JE, Song X, Akdemir ZC, Rosenfeld JA, Brown CW, et al. Phenotypic expansion of POGZ-related intellectual disability syndrome (White-Sutton syndrome). Am J Med Genet A. 2020;182(1):38-52.

13. Zhytnik L, Maasalu K, Pashenko A, Khmyzov S, Reimann E, Prans E, et al. COL1A1/2 Pathogenic Variants and Phenotype Characteristics in Ukrainian Osteogenesis Imperfecta Patients. Front Genet. 2019;10:722.

14. Overwater E, Efrat R, Barge-Schaapveld D, Lakeman P, Weiss MM, Maugeri A, et al. Autosomal dominant Marfan syndrome caused by a previously reported recessive FBN1 variant. Mol Genet Genomic Med. 2019;7(2):e00518.

15. Kang H, Aryal ACS, Marini JC. Osteogenesis imperfecta: new genes reveal novel mechanisms in bone dysplasia. Transl Res. 2017;181:27-48.

16. Swinnen FK, Coucke PJ, De Paepe AM, Symoens S, Malfait F, Gentile FV, et al. Osteogenesis Imperfecta: the audiological phenotype lacks correlation with the genotype. Orphanet J Rare Dis. 2011;6:88.

17. Gutmann DH, Ferner RE, Listernick RH, Korf BR, Wolters PL, Johnson KJ. Neurofibromatosis type 1. Nat Rev Dis Primers. 2017;3:17004.

18. Karkinen J, Miettinen PJ, Raivio T, Hero M. Etiology of severe short stature below - 3 SDS in a screened Finnish population. Eur J Endocrinol. 2020;183(5):481-8.

19. Valenza F, Cittaro D, Stupka E, Biancolini D, Patricelli MG, Bonanomi D, et al. A novel truncating variant of GLI2 associated with Culler-Jones syndrome impairs Hedgehog signalling. PLoS One. 2019;14(1):e0210097. 
20. Bougeard G, Hadj-Rabia S, Faivre L, Sarafan-Vasseur N, Frebourg T. The Rapp-Hodgkin syndrome results from mutations of the TP63 gene. Eur J Hum Genet. 2003;11(9):700-4.

21. Derbent M, Oncel Y, Tokel K, Varan B, Haberal A, Yazici AC, et al. Clinical and hematologic findings in Noonan syndrome patients with PTPN11 gene mutations. Am J Med Genet A. 2010;152A(11):2768-74.

22. Lavillaureix A, Heide S, Chantot-Bastaraud S, Marey I, Keren B, Grigorescu R, et al. Mosaic intragenic deletion of FBN2 and severe congenital contractural arachnodactyly. Clin Genet. 2017;92(5):556-8.

23. Callewaert BL, Loeys BL, Ficcadenti A, Vermeer S, Landgren M, Kroes HY, et al. Comprehensive clinical and molecular assessment of 32 probands with congenital contractural arachnodactyly: report of 14 novel mutations and review of the literature. Hum Mutat. 2009;30(3):334-41.

24. Morel Swols D, Foster J 2nd, Tekin M. KBG syndrome. Orphanet J Rare Dis. 2017;12(1):183.

25. Slaney SF, Oldridge M, Hurst JA, Moriss-Kay GM, Hall CM, Poole MD, et al. Differential effects of FGFR2 mutations on syndactyly and cleft palate in Apert syndrome. Am J Hum Genet. 1996;58(5):923-32.

26. Brandom BW. The genetics of malignant hyperthermia. Anesthesiol Clin North Am. 2005;23(4):615-9, viii.

27. Balci TB, Hartley T, Xi Y, Dyment DA, Beaulieu CL, Bernier FP, et al. Debunking Occam's razor: Diagnosing multiple genetic diseases in families by wholeexome sequencing. Clin Genet. 2017;92(3):281-9.

28. Ye X, Fang D, He Y, Yan H, Qiu W, Sun Y. Dual diagnosis of osteogenesis imperfecta (OI) and short stature and advanced bone age with or without earlyonset osteoarthritis and/or osteochondritis dissecans (SSOAOD) reveals a cumulative effect on stature caused by mutations in COL1A1 and ACAN genes. Eur J Med Genet. 2020;63(12):104074.

29. Xue Y, Ankala A, Wilcox WR, Hegde MR. Solving the molecular diagnostic testing conundrum for Mendelian disorders in the era of next-generation sequencing: single-gene, gene panel, or exome/genome sequencing. Genet Med. 2015;17(6):444-51.

30. Wu N, Ming X, Xiao J, Wu Z, Chen X, Shinawi M, et al. TBX6 null variants and a common hypomorphic allele in congenital scoliosis. N Engl J Med. 2015;372(4):341-50.

31. Liu J, Wu N, Deciphering Disorders Involving S, study CO, Yang N, Takeda K, et al. TBX6-associated congenital scoliosis (TACS) as a clinically distinguishable subtype of congenital scoliosis: further evidence supporting the compound inheritance and TBX6 gene dosage model. Genet Med. 2019;21(7):1548-58.

32. Chen W, Lin J, Wang L, Li X, Zhao S, Liu J, et al. TBX6 missense variants expand the mutational spectrum in a non-Mendelian inheritance disease. Hum Mutat. 2020;41(1):182-95.

33. Lin M, Liu Z, Liu G, Zhao S, Li C, Chen W, et al. Genetic and molecular mechanism for distinct clinical phenotypes conveyed by allelic truncating mutations implicated in FBN1. Mol Genet Genomic Med. 2020;8(1):e1023.

34. Tian W, Huang Y, Sun L, Guo Y, Zhao S, Lin M, et al. Phenotypic and genetic spectrum of isolated macrodactyly: somatic mosaicism of PIK3CA and AKT1 oncogenic variants. Orphanet J Rare Dis. 2020;15(1):288.

35. Wang K, Zhao S, Liu B, Zhang Q, Li Y, Liu J, et al. Perturbations of BMP/TGF-beta and VEGF/VEGFR signalling pathways in non-syndromic sporadic brain arteriovenous malformations (BAVM). J Med Genet. 2018;55(10):675-84.

36. Wang K, Zhao S, Zhang Q, Yuan J, Liu J, Ding X, et al. Whole-exome sequencing reveals known and novel variants in a cohort of intracranial vertebralbasilar artery dissection (IVAD). J Hum Genet. 2018;63(11):1119-28.

37. Chen N, Zhao S, Jolly A, Wang L, Pan H, Yuan J, et al. Perturbations of genes essential for Mullerian duct and Wolffian duct development in MayerRokitansky-Kuster-Hauser syndrome. Am J Hum Genet. 2021.

38. Richards S, Aziz N, Bale S, Bick D, Das S, Gastier-Foster J, et al. Standards and guidelines for the interpretation of sequence variants: a joint consensus recommendation of the American College of Medical Genetics and Genomics and the Association for Molecular Pathology. Genet Med. 2015;17(5):40524.

\section{Figures}




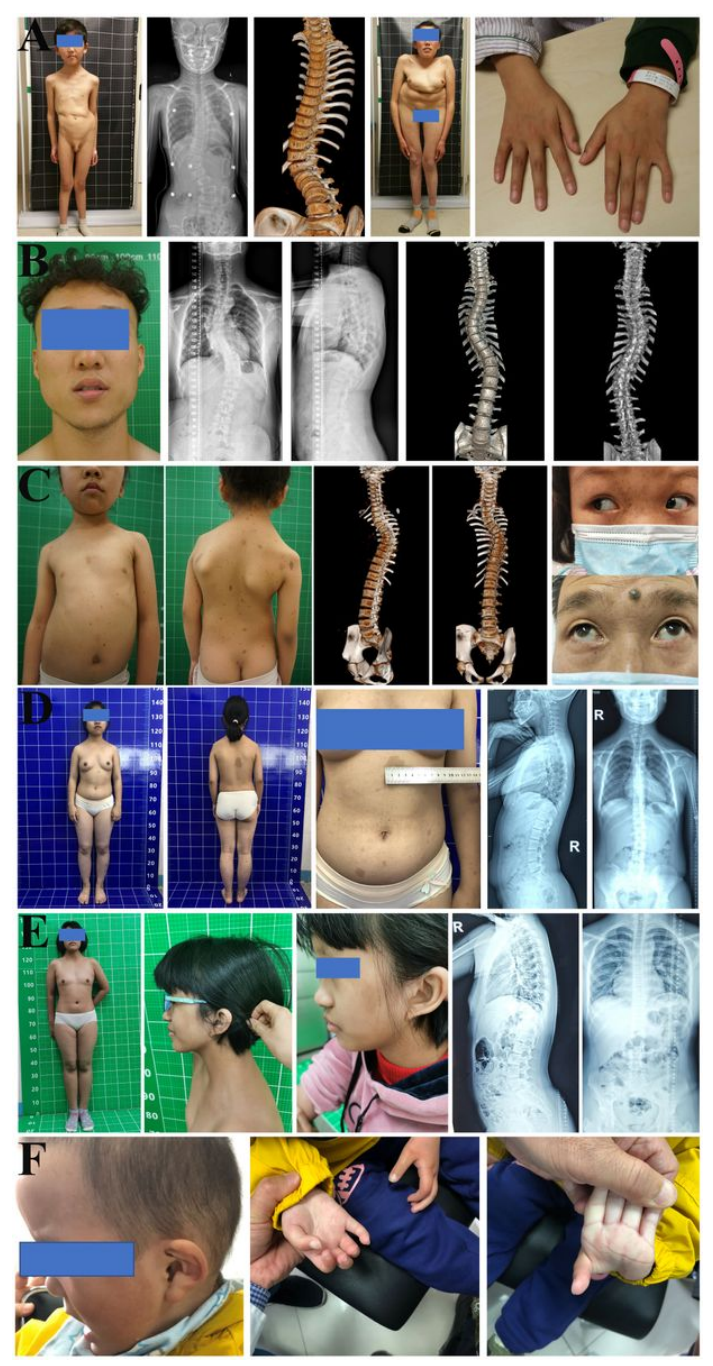

\section{Figure 1}

Representative clinical photographs of 6 patients with skeletal abnormality. A Patient DISCO-SC02003P1972. B Patient DISCO-SC01908P0067. C Patient DISCO-PCT2007P0019. D Patient DISCO-AX168. E Patient DISCO-AX282. F Patient DISCO-RDD2001P0005

\section{Supplementary Files}

This is a list of supplementary files associated with this preprint. Click to download.

- Supplementaryfigure1.jpg 